\title{
ON SUBJUNCTIVE CLASSES OF GROUPS
}

\author{
Javier Dtal
}

Throughout, the terminology is that of [5]. A class $\underline{x}$ of groups is said to be subjunctive if it is $\$_{n}$ and $N_{0}-c$ losed, that is if subnormal subgroups of $X$-groups and groups generated by finitely many normal $\underline{x}$-subgroups are in $\underline{x}$. These classes were introduced in [6] for the study of the subnormal structure of a group and have al so been considered under the point of view of the dualization of Gaschitz's formation theory of finite soluble groups $([1],[3])$. In the present paper we shall deal with a classification of subjunctive classes in two disjoint types, as the following result shows

Theorem A subjunctive class either only contains perfect groups having no non-trivial cyctic subnormal subgroups or contains every finite p-group, for some prime $p$.

Its proof needs an auxiliary result which we state in a more general form because of its own interest. We recall that an $\mathrm{N}_{1}$-group is a group in which every subgroup is subnormal; this class is not. $D_{0}$-closed and we have

Lemma Let $\underline{X}$ be a subjunctive class. If $\underline{Y}$ is a $D_{0}$-closed subelass of $N_{1}$-groups then every $\underline{Y}$-group is nitpotent and $H \underline{X} \cap \underline{Y} \leq \underline{X}$.

Proof: First, let $G \in \underline{Y}$; then $G \times G \in \underline{Y}$ and, in particular, the diagonal subgroup $D$ of $G \times G$ is subnormal. If $D=D_{0} \circ D_{0} \diamond \ldots \Delta D_{r}=G \times G$ is a subnormal chain from $D$ to $G \times G$ and $z_{j}$ is the $i^{\text {th }}$-term of the upper central series of $G$ we obtain by an easy induction that if $(x, y) \in D_{i}$ then $x Z_{i}=$ $=y z_{i}$. Therefore $G=z_{r}$ and then $G$ is nilpotent.

Now, let $G$ be an $\underline{X}$-group with a normal subgroup $N$ such that $G / N \in \underline{Y}$. 
Let $\left\{Z_{i} / N \mid 0 \leq i \leq c\right\}$ be the, upper central series of $G / N$; in order to show that $G / N \in \underline{X}$, we may assume that $Z_{i} / N \in \underline{X}$ if $i<c$. Let $K=G \times(G / N)$ and denote by $G_{0}$ the image isomorphic to. $G_{\text {. in }} K$ under the map that sends $x$ to $\left(x_{2} \times N\right)$. If $K_{j}=Z_{i} \times\left(Z_{j} / N\right)$ then $k_{i} \in \underset{\sim}{X}$ if $i<c$ and, since $k_{j-l} G_{0}$ is normal in $K_{i} G_{0}$, we have by an easy induction that $K_{i} G_{0} \in X_{1}$ if $j<c$ But $K=(G \times l) G_{0}=(G \times H)\left(K_{C-1} G_{0}\right) \in \times$ and then $G / N<X$.

Proof of Theorem: Let $\underline{x}$ be a subjunctive class. Denote by. $\underline{y}$ the class of perfect groups having no non-trivial cyclic subnormal subgroups and assume that $\underline{X}$ is not contained in $\underline{U}$. Let $I \neq G \in \underline{X}$. If $G$ is perfect then it must contain a non-trivial cyclic subnormal subgroup, which necessarily belongs to $\underline{X}$. If $G^{\prime}$ is proper then $G / G^{\prime} E \underline{X}$ by Lemua and each one of its non-trivial cyclic subgroups belongs to $\underline{x}$. Thus $\underline{x}$ contains a nontrivial cyclic group, say $H$. If $H$ is finite then there exists a prime $p$ such that $G$ contains a cyclic subgroup of order $p$, which necessarily belongs to $x$. If $H$ is infinite then for every prime $p$ the group $H / H^{p}$ has order $p$ and belongs to $\underline{x}$ by Lemma. Therefore, in any case, $\underline{X}$ contains $a$ copy of a cyclic group of order $p$, for some prime $p$. Since any finite p-group $P$ can be subnormally embedded in a finite group $Q$ generated by subnormal subgroups of order $p$ (see [3] p. 204) it follows from the $f i$ niteness of $Q$ that $Q . X \underline{X}([1] \times .1 . d)$ and then $P \in \underline{X}$, as desired.

Classes of the second type are very common and it is possible to enlarge the stock of finite nilpotent groups contained in a such class obtainning similar results to those of [3] 0.204 ; sometimes that fact depends on the kind of cyclic groups contained in $\underline{x}$; for example if $\underline{x}$ contains an infinite cyclic group then every finite nilpotent group belongs to $\underline{X}$. On the other hand, $P$. Hall [2] produced examples of characteristically simple groups with trivial Baer radical; such groups are in $\underline{U}$. It is also clear that a non-abelian simple group is an U-group; 
since $\underline{U}$ is $P$ and $D$-closed it can be proved that if $\underline{Y}$ is a class of such groups then $D_{0} Y, D \underline{Y}$ and the radical class generated by $\underline{Y}$ are subjunct $i$ ve classes of $\mathrm{U}$-groups. These groups can be taken to be torsion-free, periodic or mixed (see [4]).

We can apply our result in order to give a negative answer to the $\mathrm{N}_{0}$-closure of certain classes of groups. For example Corollary 1 The following classes are not $N_{0}$-closed: (i) The class of groups with trivial Baer radical; (ii) Any s-ctosed class of torsionfree groups; (iii) A proper and non-trivial quasivariety of groups. Proof: (i) Such class contains U properly. (ii) Every $\$$-closed subjunctive class must contain a group of order $p$. (iii) Let $\underline{x}$ be a nontrivial quasivariety, that is $S$ and $R$-closed. If $\underset{\mathcal{X}}{X}=N_{0} \underset{X}{X}$, as above, $\underline{X}$ contains every finite $p$-group, for some prime $p$, and then we may deduce that every group is in $\underline{X}$ (Apply [5] 9.11 and 8.19.2).

In contrast with (ii) we have already seen that there are subjunctive classes of U-groups consisting of torsion-free groups. He also remark that (iii) can be applied to varieties of groups; since a variety is $\mathrm{H}$-closed then it is never P-closed, except for trivial cases. However there exist proper and non-trivial P-closed quasivarieties of groups.

Finally, if $\underline{Y}$ is a class then the class $L_{n} Y$ is defined to be the ciass of all groups in which every finitely generated subgroup is contained in some subnormal $\underline{Y}$-subgroup. In [6] it was studied the behaviour of this operator with respect to $\mathrm{N}_{0}$-closed classes. Joinning [6] Theorem $C$ to our Theorem we readily obtain Corollary 2 If $\underline{\underline{Z}}$ is a subjunctive class then $L_{n} \underline{X}=N \underline{X}$ is an $N$-closed subjunctive class of the same type.

Remark that, as a consequence, we have just proved that if $x$ is contained in $\underline{U}$ then $\underline{U}$ contains $\mathbf{N} \underline{X}$ as well. 
Acknowledgement. The author wishes to thank the referee for pointing out the present version of Lema on the first page of this paper.

\section{REFERENCES}

[1] W. Gaschütz. Lectures on subgroups of Sylow type in finite soluble groups. Notes on Pure Mathematics, 11. Australian National University, Canberra 1979.

[2] P. Hall. Wreath products and characteristically simple groups. Proc, Camb. Phil. Soc. 58 (1962), 170-184.

[3] B. Hartley. On Fischer's dualization of Formation Theory. Proc. London Math. Soc. (3) 19 (1969), 193-207.

[4] A. Ju. 01'sanskit. Infinite groups with cyclic subgroups. Soviet Math. Doklady 20 (1979), 343-346.

[5] 0. J. S. Robinson. Finiteness conditions and generalized soluble groups. Springer-Verlag, Berlin 1972.

[6] J. E. Roseblade and S. E. Stonehewer. Subjunctive and Locally Coalescent classes of groups. J. Algebra 8 (1968), 423-435.

Rebut el 15 de febrer del 1985

Facultad de Matemáticas

Departamento de Algebra

Universidad de Zaragoza

50009 zaragoza

SPAIN 